\title{
COMPARING IMPLANT SUPPORTED MANDIBULAR OVERDENTURE WITH CONVENTIONAL COMPLETE DENTURE REGARDING OCCLUSION AND DISOCCLUSION TIME USING T SCAN
}

\author{
Mahmoud El Moutassim-Bellah El Homossany* and Hebatallah Tarek Abdallah*
}

\begin{abstract}
Aim of the study: to compare complete denture with implant supported overdenture regarding occlusion and disocclusion time using a $\mathrm{T}$ scan.

Materials and methods: A crossover clinical trial was adopted,50 patients were selected for this study. The assessment was carried out with conventional complete dentures and then with the same dentures after loading of the implants. Computerized occlusal analysis using the T-Scan III system was conducted to perform baseline computer-guided occlusal adjustment for conventionally fabricated dentures. Recording of OT (occlusion time) and DT (Disocclusion time) right and left values using the T-Scan were subsequently carried out for all dentures, first without implants and then after implants loading. All T-Scan procedures were carried out by the same clinician.
\end{abstract}

Results: Independent $t$ test was used in this study because data was normally distributed. The OT values were recorded and a mean OT value was calculated for each subject, where the CD Complete denture group mean OT $(0.49 \pm(0.21) \mathrm{s}$ which was about $0.13 \mathrm{~s}$ longer than the Implant Supported Over Denture(ISOD) mean OT $(0.36 \pm(0.19)$. The disocclusion time were for right $0.55 \pm$ 0.25 for $C D$ and $0.35 \pm 0.32$ for ISOVD, while on the left was $0.56 \pm 0.24$ for CD and $0.37 \pm 0.27$ for ISOVD. There were highly significant results between the two groups in Occlusion time and disocclusion RT and left $0.0016,0.0007,0.0003$ respectively.

Conclusion: Using the computerized analysis of the Occlusion Time, Implant supported over denture proved to be a better treatment option regarding occlusion and disocclusion time where timing decreased significantly after implant loading.

\section{INTRODUCTION}

A recording medium is necessary to register the patient's interarch relationship. If the interarch registration is inaccurate, the mounted casts will not show the patient's existing maxillomandibular relationship, and errors in diagnosis and treatment will result.

The introduction of different interocclusal recording materials has put clinicians in dilemma. In the era of developing world of dentistry the

* Lecturer Removable Prosthodontics Department, Faculty of Dentistry, Ain Shams University. 
different materials are introduced for interocclusal record with different brand names because of this; the utility of the material is confusing for successful delivery of prosthesis. ${ }^{(1)}$

Occlusal forces that can destabilize a complete denture prosthesis during function can result from a poor occlusal force summation location ${ }^{(2,3)}$. This unseen collection of unbalanced forces can assist in movement and dislodgment of both upper and lower opposing complete dentures, while the prostheses are subjected to occlusal function. Developing a complete denture occlusion that aligns the occlusal forces along the posterior midline of the hard palate and within the center of the distribution of occlusal contacts will insure the denture is seated vertically during occlusal loading upon the most supportive edentulous tissue-bearing area.

Limitations during registration and transference stages of the maxillomandibular relationship are encountered due to a variety of difficult intraand extraoral conditions, resulting in some errors in the interocclusal relationship of mounted casts. ${ }^{(4)}$ One of the causes of this occlusal inaccuracy attributable to the clinical stage of interocclusal registration is related to the properties of the interocclusal recording materials ${ }^{(5,6)}$ because they can critically affect the accuracy of the interocclusal registration, apart from the operator's clinical ability and the techniques followed. ${ }^{(7,8)}$ Among the properties of an ideal interocclusal recording material, the most important parameter that may be used for selection of the material is dimensional stability and accuracy because this avoids any discrepancies between the maxillomandibular registration and mounting of the casts. ${ }^{(7)}$

Occlusal contact is usually assessed with articulating paper to reveal initial contacts. Dark, wide marks are understood to be sites of high pressure, whereas lighter marks are associated with sites subjected to lesser loads, and the presence of numerous, similar markings is suggestive of equal, evenly distributed loads. However, as some authors have noted, the marks produced by articulating paper may be difficult to reproduce and lend themselves to subjective interpretation. A study designed to investigate marking surface as the only variable, articulating paper was placed between epoxy resin models of dental arches that were then mounted on an articulator and subjected to compression loads ranging from 25-450 Newtons. The results showed that despite a general increase in the marking surface with an increase in applied load, there was only a one-in-five chance that a pair of similar markings would describe the same load. Moreover, because articulating paper is unable to register occlusal contact force or time sequencing, it is an insufficient indicator of occlusal contact simultaneity. ${ }^{(10,11)}$

The objective assessment of occlusal equilibration has been possible in recent years through computerized occlusal analysis. The T-Scan system was developed by Maness et $\mathrm{al}^{(12)}$ in 1987. The latest version (T-Scan III) provides a dynamic visual evaluation of a patient's occlusion from initial tooth contact to maximum intercuspation. ${ }^{(13-15)}$ The system records relative force values ${ }^{(16)}$ but it allows for objective quantitative evaluation of occlusal balance by recording timed occlusal contacts and by displaying numerical values for occlusion and disocclusion times. Occlusion time (OT) is the time from the first contact of occluding teeth to maximum intercuspation, and disocclusion time (DT) is the time from maximum intercuspation to complete disocclusion during lateral movement. ${ }^{(17-19)}$ The clinical goal is to reach maximum intercuspation in less than 0.2 second and disocclude all posterior teeth in less than 0.4 second. ${ }^{(20)}$ The shorter the OT, the less time required to contact all teeth and the fewer the prematurities as the patient closes into maximum intercuspation, indicating occlusal contact simultaneity and equilibration. Shorter DT (less than 0.5 second) has shown to decrease contractile muscle activity significantly to near resting state values during mandibular excursions 
and decrease the stress on the temporomandibular joints (TMJs). ${ }^{(20-22)}$

The T-Scan system was found to be considerably accurate and reliable ${ }^{(22-25)}$ and has been implemented in different clinical dental applications, ${ }^{(26-31)}$ including complete denture prosthodontics. ${ }^{(32,33)}$ Of those studies, only 1 investigated both OT and DT in complete denture wearers. ${ }^{(17)}$

A study was made where FGP functional generating path single crown was made versus normal occlusion. The results showed significantly smaller changes in OT and DT than those from conventional single crowns.

\section{MATERIALS AND METHODS}

A crossover clinical trial was used. The evaluation was carried out with conventional complete dentures and then with the same dentures after implant placement.

Edentulous participants, (50 to 62 years of age) were selected from the Department of Oral and Maxillofacial Prosthodontics at Ain Shams University. All patients were given detailed information about the investigation and provided written informed consent for their participation. All participants had an Angle class I jaw relationship with no history of temporomandibular disorders. The ridges were well developed, and the mucosa was healthy and firmly attached to the underlying bone.

Exclusion criteria included the presence of major medical problems or severe chronic diseases or any intraoral soft or hard tissue pathology. New conventional maxillary and mandibular complete dentures were fabricated for all participants in a conventional manner.

Elastomeric definitive impressions with border tracing were made in custom trays. Maxillomandibular records were made using rubber base; vinyl polysiloxane as interocclusal records material* and mounted on semi-adjustable articulators using facebow **, centric relation, and protrusive records. The occlusal scheme was bilaterally balanced occlusion. Laboratory remounting was provided to correct occlusal errors. Clinical adjustments were then carried out conventionally on the day of denture insertion and continued throughout the first week until participants were free from any discomfort from their dentures. Clinical remounting procedures were then carried out two weeks later to refine occlusal contacts after denture settling.


Fig. (1) Bite registration for the patient using vinyl polysiloxane

\footnotetext{
* Kettenbach GmbH\&Co.KG,Germany

** Dentatus AB Jakobsdalsvagen Ha,gersten,Sweden
} 


\section{T-Scan device*** : a T-Scan device used for occlusal load equilibration}

It consists of :

- Scan handle and lab-top.

- Box of large and box of small sensors.

- Box of large and box of small sensor supports.

- The sensor support provided enough width to cover the molars and the patients were allowed to close comfortably, tried in to choose the correct support size.

Sensors support was attached to the T-Scan handle with the "Sensor Position Guide" faced up until it clicks into place.

- The latch was lifted, and the sensor was inserted, which were the same size as support so that the outside edges of the sensor slid into the sensor support channels then the latch was closed to lock the center in place. A green light indicated that the sensor was properly connected.

\section{- Patient Record:}

Records done in centric, eccentric and protrusive positions by the following steps:

- The patient record was created by clicking "New Patient," and the patient information, first, middle and last name, gender, date of birth and ID number was filled.

- The sensor was inserted into the mouth like that in impression tray.

- Patient opened his/her mouth slightly.

- The sensor was inserted so that the position guide touch the central incisor at the midline.

- The sensor was held in place so that the sensor level with the occlusal plan.

- T-Scan device used a range of colors to differentiate the forces on the teeth, Adjusting sensitivity ensure the entire color spectrum is used to visualize the differences in these forces and the maximum forces are within the sensor force range, and this was done by:

- The patient was asked to bit and clench firmly

- The patient was asked to clench, and the tooth contact was observed on the screen.

- The ideal setting is maximum three pink contact.

- Records taken at centric, eccentric and protrusive positions.

Before measuring the timing, corrective occlusal adjustments were carried out using the center of force (COF) concept for each participant. Each patient was asked to firmly occlude into the sensor with their dentures until maximum intercuspation occurred, maintaining their teeth together for 1 to 3 seconds, and then disoccluding and re intercuspating into the sensor once again. Premature contacts were then adjusted based on the overloaded contact locations displayed and demarcated within the system's 2D and 3D force/view panes (through color coding and force values).

Each reading was repeated four times, and an average reading was taken for each patient for OT and D T.

All patients later were prepared to receive two conventional $3.25 \mathrm{~mm}$ diameter and $10 \mathrm{~mm}$ length * implants in the interforaminal region of mandibular ach.

The lower denture was duplicated and used to construct clear acrylic surgical stent, then the proposed sites of the implants were marked on the surgical stent and holes have been drilled The Surgical stent was used to guide the insertion of the two implants in the anterior area between the two mental foramina.

*** Tekscan $^{\circledR}$, south Boston, U.S 
After flab reflection, $1.3 \mathrm{~mm}$ pilot drill was lightly pumped up and down vertically perpendicular to the occlusal plane through the holes of the surgical stent, penetrating the crestal bone 3-4 $\mathrm{mm}$. Sterile internal and external saline irrigation was used throughout the drilling procedure

The pilot holes were deepened to a depth of the implants to be placed using osteotomy drill of $10 \mathrm{~mm}$. Implant $3.25 \mathrm{~mm}$ diameter, $10 \mathrm{~mm}$ length* was carried to the osteotomy site, and was rotated in a clockwise direction while exerting a slight downward pressure using a finger ratchet driver.

The wrench torque was connected to ratchet driver (with the directional arrow facing a clockwise).

The final stage of placement was carried out with carefully controlled turns for full seating, 1/4 turn rotation with 15 seconds pauses between rotations .once implants is in place, cover screw are added until delayed loading.

After three months, abutments were placed, and metal housing was picked up in the fitting surface of the denture, and a new Tscan record was made after loading of the implants.

Statistical analysis was performed with IBM $^{\circledR}$ SPSS $^{\circledR}$ Statistics Version 20 for Windows. Data were collected, tabulated, and presented as means, standard deviations, and medians. The KolmogorovSmirnov test of normality was conducted for the OT, DT right and left data. Most of the variables were found to be normally distributed. Data were, therefore, statistically analyzed with the paired t test $(\mathrm{p}=0.0005)$.



Fig. (2) T scan device and sensors

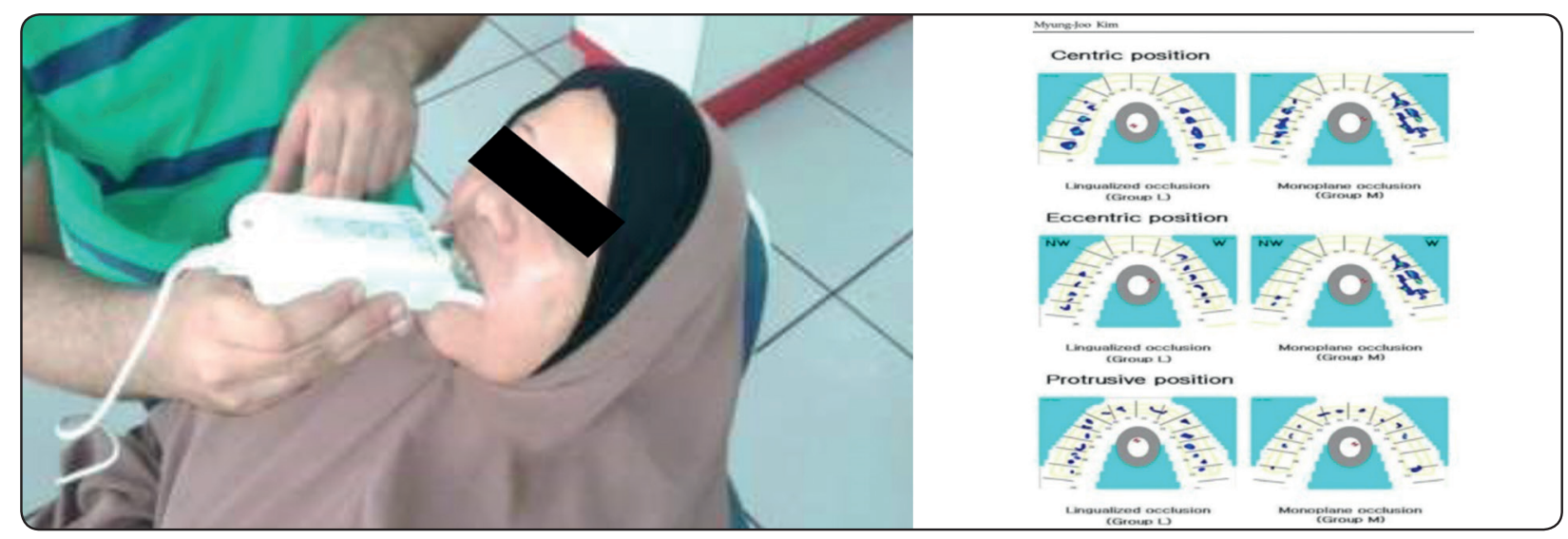

Fig (3) Recording the timing of occlusion and disocclusion and examples of computer displays of T scan

\footnotetext{
* INNO fixture,Cowellmedi.Co.,Ltd,German

${ }^{\circledR}$ IBM Corporation, NY, USA.

${ }^{\circledR}$ SPSS, Inc., an IBM Company.
} 


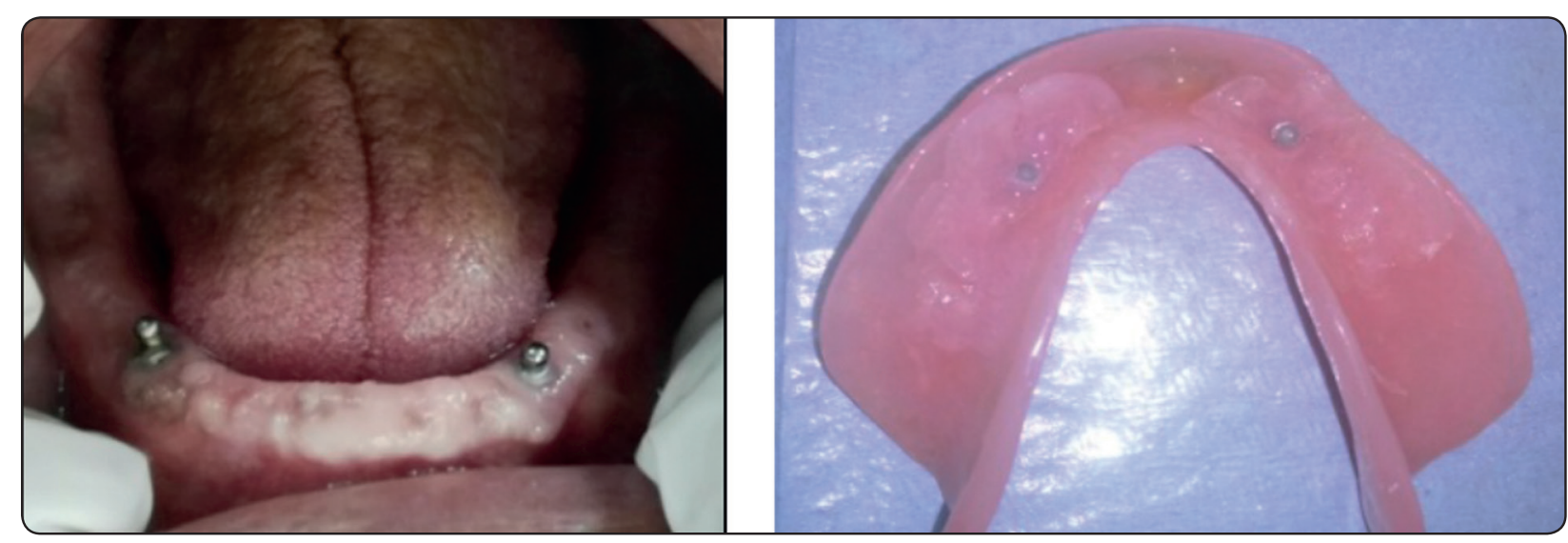

Fig (4) Implants intra oral view and intalgio view of the denture

\section{RESULTS}

A total of 50 subjects included in the study underwent the recording of their occlusion times during Mandibular opening- closing movements. The OT values were recorded and a mean OT value was calculated for each subject, where the $\mathrm{CD}$ group mean OT $(0.49 \pm(0.21) \mathrm{s})$ was about $0.13 \mathrm{~s}$ longer than the ISOVD mean OT $(0.36 \pm(0.19)$. This was of statistical significance.

The disocclusion time were for right $0.55 \pm(0.25)$ for $C D$ and $0.35 \pm(0.32)$ for ISOVD , while on the left was $0.56 \pm(0.24)$ for $\mathrm{CD}$ and $0.37 \pm(0.27)$ for ISOVD

There was highly significant results between the two groups in Occlusion time and disocclusion RT and left $0.0016,0.0007,0.0003$ respectively.

\section{DISCUSSION}

One of the causes of occlusal inaccuracy is attributed to the clinical stage of interocclusal registration. So proper choice was made for the interocclusal recording material in this study. vinyl Polysiloxane was used because the material has high dimensional stability. ${ }^{(7)}$

The computerized occlusal analysis was conducted using the T-Scan system. The system uses a $100 \mathrm{~mm}$ thick recording sensor that scanned in 0.003 second increments. Occlusal adjustment for all dentures was made first using $\mathrm{T}$ scan. It was then used to record occlusal parameters, namely, OT and DT, for dentures without and with Implants. All scanning procedures were carried out by the same clinician. The size of the sensor (large or small) was chosen to suit the participant's dental

TABLE (1) Occlusion and disocclusion time (seconds) in conventional versus implant supported overdenture.

\begin{tabular}{|c|c|c|c|}
\hline Occlusion parameter & $\begin{array}{c}\text { Conventional dentures } \\
\text { Mean }( \pm \mathrm{SD})\end{array}$ & $\begin{array}{c}\text { Implant supported overdenture } \\
\text { Mean }( \pm \mathrm{SD})\end{array}$ & P value \\
\hline Occlusion time & $0.49 \pm(0.21)$ & $0.36 \pm(0.19)$ & 0.0016 \\
\hline Disocclusion time Rt & $0.55 \pm(0.25)$ & $0.35 \pm(0.32)$ & 0.0007 \\
\hline Disocclusion time Lt & $0.56 \pm(0.24)$ & $0.37 \pm(0.27)$ & 0.0003 \\
\hline
\end{tabular}


arch. Before any occlusal data acquisition and according to the manufacturer's recommendation, a proper sensitivity range was established, ${ }^{(35)}$ and sensor conditioning procedures of 4 test closures were performed for each patient. For all scanning procedures, participants were requested to sit in an upright position. The sensor was held consistently in the same place concerning the teeth. It was adjusted to be parallel to the occlusal plane and centered on the midline between the central incisors ${ }^{(22)}$

Exact analysis of occlusal contacts and occlusal force is a problem in functional diagnostics that has not yet been thoroughly settled, despite the fact that the harmful consequences of an unbalanced occlusion are severe. In clinical practice, the present-day analysis of the occlusion is reduced to representing force with color-marking articulating papers that leave ink marks upon the teeth. However, these marks only indicate the localization of contacts, but do not describe reliably the occlusal force relationships. The precise analysis that incorporates time resolution and plots the distribution of forces within the occlusion is not possible when employing the traditional occlusal indicator methods. A detailed occlusal force and timing analysis can only be provided by performing a computer-assisted analysis, using the T-Scan III system which records changing relative occlusal force levels and real-time occlusal contact sequence data with High Definition (HD) recording sensors. This study demonstrates the use of this computer-based occlusal measurement method that reliably describes the time-dependent distribution of occlusal force evolution to compare between Conventional complete denture and implant supported overdenture. ${ }^{(36)}$

The significance of the occlusion time is based on a measure of the degree of the bilateral simultaneous contacts. It is known that in subjects with their natural dentition, teeth can occlude simultaneously in 0.1 seconds or less and that all teeth can measurably disocclude immediately in less than 0.3-0.5 seconds. ${ }^{(37)}$ The closer to 0.1 second that passes from the first to the last contact, the better the right side to left side, and the better the anteroposterior simultaneity is. So, the overall occlusal force balance is consequently distributed throughout the arch in a better way. This result indicates that the adaptation to the occlusal design occurs and muscular responses to the masseters and the temporalis are considerably improved ${ }^{(35)}$

The T-scan was adopted in the present study to detect occlusal contacts because it could not only locate premature contacts and occlusal interference more clearly ${ }^{(15,38)}$ but also relate occlusion to other elements of the articulatory system simply through occlusion time OT and disocclusion time DT. OT is directly related to patients' occlusal contact pattern and has been considered as a capable description of occlusion ${ }^{(39,40)}$, whereas, DT could relate tooth contacts to muscle activity ${ }^{(41)}$. Abnormalities in DT would result in a change of muscle activity, thus facilitating the occurrence of $\mathrm{TMD}^{(20)}$. According to the manufacturer, OT is recommended as less than $0.2 \mathrm{~s}$, and DT less than $0.4 \mathrm{~s}^{(35,42)}$. However, in a survey conducted by Haralur et al..$^{(19)}$, the average OT and DT in normal dentate subjects with healthy TMJs were $0.69 \mathrm{~s}$ and $0.79 \mathrm{~s}$ respectively. Another survey performed by Ma et al..$^{(43)}$ in a Chinese population with Angle class I relationship and healthy TMJs showed an average of $0.34 \mathrm{~s}$ for OT and $1.00 \mathrm{~s}$ for DT. Also, in the present study, the average OT were $0.49 \pm(0.21)$ for complete denture .0 .36 for ISOVD respectively. The disocclusion time were for right $0.55 \pm(0.25)$ for $\mathrm{CD}$ and $0.35 \pm(0.32)$ for ISOVD, while on the left was $0.56 \pm(0.24$ for $C D$ and $0.37 \pm(0.27$ for ISOVD which were between the results obtained by Haralur et al. and Ma et al., but still much longer than the recommended values. These discrepancies may be probably explained by individual difference. The phenomenon indicated that more physiologic harmonious function might be achieved by ISOVD than a complete denture. 
To ensure valid and reliable results, the occlusal adjustment and the recording of T-scan were carried out by the same examiner to exclude interexaminer variations $^{(44)}$. Furthermore, considering an increasingly significant relationship was reported between the sagittal plan head-neck posture and initial occlusal contacts for patients over the age of $30^{(45)}$, all the occlusal adjustment procedures were done with participants sitting in a relaxed upright position. As instructed by the manufacturer, the sensors of $\mathrm{T}$ scan can be used up to 15 to 25 times $^{(46)}$.

The results of OT and DT rejected the null hypothesis and revealed that OT and DT were significantly smaller in ISOVD than complete denture, Moreover, more comfortable feeling and less chairside time in ISOVD, compared with conventional dentures which was consistent with the result of a previous study carried out by adhesive versus non adhesive. ${ }^{(44)}$

\section{CONCLUSION}

Using the computerized analysis of the Occlusion Time, Implant supported overdenture proved to be a better treatment option regarding occlusion and disocclusion time where timing decreased significantly after implant loading.

\section{REFERENCES}

1. Sampath Kumar Tejo, Anil G Kumar,Vivekanand S Kattimanir, Priti D Desai, Sande P Nalla AD Krishna Chaitanya K.A comparative evaluation of dimensional stability of three types of interocclusal recording materialsan in-vitro multi-centre study. Head \& Face Medicine2012

2. Dawson PE. Diagnosis and treatment of occlusal problems. 2. St. Louis: CV Mosby Co; 1989.

3. Dawson PE. Functional occlusion from TMJ to smile design. St Louis: CV Mosby Co; 2007.

4. Freilich MA, Altieri JV, Wahle JJ. Principles for selecting interocclusal records for articulation of dentate and partially dentate casts. J Prosthet Dent 1992;68:361-7.

5. Balthazar-Hart Y, Sandrik JL, Malone WFP, Mazur B, Hart T. Accuracy and dimensional stability of four interocclusal recording materials. J Prosthet Dent 1981;45:586-91.
6. Michalakis KX, Pissiotis A, Anastasiadou V, Kapari D. An experimental study on particular physical properties of several interocclusal recording media. Part I: consistency prior to setting. J Prosthodont 2004;13:42-6.

7. Michalakis KX, Pissiotis A, Anastasiadou V, Kapari D. An experimental study on particular physical properties of several interocclusal recording media. Part II: linear dimensional change and accompanying weight change. J Prosthodont 2004 Sep;13(3):150-9

8. Chen SY, Liang WM, Chen FN. Factors affecting the accuracy of elastometric impression materials. J Dent 2004;32:603-9.Prosthodont 2004;13:150-9.

9. Cohen-Levy J, Cohen N. Computerized analysis of occlusal contacts after lingual orthodontic treatment in adults. Int Orthod 2011;9:410-431.

10. Kerstein RB. Articulating paper mark misconceptions and computerized occlusal analysis technology. Dent Implantol Update 2008;19:41-46.

11. Carey JP, Craig M, Kerstein RB, Radke J. Determining a relationship between applied occlusal load and articulating paper mark area. Open Dent J 2007;1:1-7.

12. Maness WL, Benjamin M, Podoloff R, Bobick A, Golden RF. Computerized occlusal analysis: a new technology. Quintessence Int 1987;18:287-92.

13. Koos B, Godt A, Schille C, Göz G. Precision of an instrumentation-based method of analysing occlusion and its resulting distribution of forces in thedental arch. J Orofac Orthop 2010;71:403-10.

14. Martins MJS, Caramelo FJ, Ramalho da Fonseca JA, Nicolau PMG. In vitro study on the sensibility and reproducibility of the new T-Scan III HD system.Rev Port Estomatol Med Dent Cir Maxilofac 2014;55:14-22.

15. Kerstein RB, Thumati P, Padmaja S. Force finishing and centering tobalance a removable complete denture prosthesis using the T- Scan III computerized occlusal analysis system. J Indian Prosthodont Soc 2013;13:184-8.

16. Throckmorton GS, Rasmussen J, Carloss R. Calibration of T-Scan sensors for recording bite forces in denture patients. J Oral Rehabil 2009;36:636-43.

17. Sierpinska T, Golebiewska M, Kuc J, Lapuc M. The influence of the occlusal vertical dimension on masticatory activities and hyoid bone position incomplete denture wearers. Adv Med Sci 2009;54:104-8. 
18. Gümüs s HÖ, Kılınç H_I, Tuna SH, Ozcan N. Computerized analysis of occlusal contacts in bruxism patients treated with occlusal splint therapy. J Adv Prosthodont 2013;5:256-61.

19. Haralur SB. Digital evaluation of functional occlusion parameters and their association with temporomandibular disorders. J Clin Diagn Res 2013;7:1772-5.

20. Kerstein RB, Wright N. Electromyographic and computer analyses of patients suffering from chronic myofascial pain-dysfunction syndrome: before and after treatment with immediate complete anterior guidance development.J Prosthet Dent 1991;66:677-86.

21. Kerstein RB. Disocclusion time-reduction therapy with immediate complete anterior guidance development to treat chronic myofacial pain-dysfunction syndrome. Quintessence Int 1992;23:735-47.

22. Kerstein RB, Radke J. The effect of disclusion time reduction on maximal clench muscle activity levels. Cranio 2006;24:156-65.

23. Saraço_glu A, Ozpinar B. In vivo and in vitro evaluation of occlusal indicator sensitivity. J Prosthet Dent 2002;88:522-6.

24. Garrido García VC, García Cartagena A, González Sequeros O. Evaluation ofocclusal contacts in maximum intercuspation using the T-Scan system. J OralRehabil 1997;24:899-903.

25. González Sequeros O, Garrido García VC, García Cartagena A. Study of occlusal contact variability within individuals in a position of maximum intercuspation using the T-SCAN system. J Oral Rehabil 1997;24:287-90.

26. Garg AK. Analyzing Dental Occlusion for implants: Tek Scan's T Scan III.Dent Implantol Update 2007;18:65-70.

27. Kerstein RB. T-scan III applications in mixed arch and complete arch,implant-supported prosthodontics. Dent Implantol Update 2008;19:49-53.

28. Montgomery MW, Shuman L, Morgan A. T-scan dental force analysis forroutine dental examination. Dent Today 2011;30:112-4. 116 .

29. Iwase M, Sugimori M, Karachi Y, Nagumo M. Changes in bite force andocclusal contacts in patients treated for mandibular prognathism by orthognathic surgery. J Oral Maxillofac Surg 1998;56:850-5.

30. Suda S, MacHida N, Momose M, Yamaki M, Seki Y, Yoshie $\mathrm{H}$, et al.A multiparametric analysis of occlusal and periodontal jaw reflex characteristics in adult skeletal mandibular protrusion before and after orthognathic surgery. J Oral Rehabil 1999;26:686-90.

31. Sierpinska T, Golebiewska M, Lapuc M. The effect of mastication on occlusal parameters in healthy volunteers. Adv Med Sci 2008;53:316-20.

32. Olivieri F, Kang KH, Hirayama H, Maness WL. New method for analyzing complete denture occlusion using the center of force concept: a clinical report.J Prosthet Dent 1998;80:519-23.

33. Boening KW, Walter MH. Computer-aided evaluation of occlusal load in complete dentures. J Prosthet Dent 1992;67:339-44.

34. Ping-ting Lin, Yang Jiao,San-jun Zhao, Fu Wang, Ling Li, Fan Yu, Min Tian, Hao-han Yu, and Ji-hua Chen Occlusion and Disocclusion Time Changes in Single Unit Crowns Designed by Functional Generated Path Technique: A Randomised Clinical Trial. Sci Rep. 2017; 7: 388.

35. Kerstein RB, Grundset K. Obtaining bilateral simultaneous occlusal contacts with computer analyzed and guided occlusal adjustments. Quintessence Int2001;32:7-18.

36. Koos, B. University Medical Center Schleswig-Holstein, Germany Medical Imaging: Concepts, Methodologies, Tools, and Applications Precision and reliability of the T-Scan III System: Analyzing occlusion and the resultant timing and distribution of forces in the dental arch18 July 2016, Pages 846-875

37. Kerstein RB. Combining technologies: acomputerized occlusal analysis system synchronized with a computerized electromyography system. Cranio. 2004Apr;22(2):96-109.

38. da Silva Martins MJ, Caramelo FJ, Ramalho da Fonseca JA, Gomes Nicolau PM. In vitro study on the sensibility and reproducibility of the new T-Scan $®$ III HD system. Revista Portuguesa de Estomatologia, Medicina Dentária e Cirurgia Maxilofacial. 2014;55:14-22.

39. Wang C, Yin X. Occlusal risk factors associated with temporomandibular disorders in young adults with normal occlusions. Oral surgery, oral medicine, oral pathology and oral radiology. 2012;114:419-423.

40. Baldini A, Nota A, Cozza P. The association between Occlusion Time and Temporomandibular Disorders. Journal of electromyography and kinesiology: official journal of the International Society of Electrophysiological Kinesiology. 2015;25:151-154 
41. Cheng HJ, Geng Y, Zhang FQ. [The evaluation of intercuspal occlusion of healthy people with T-Scan II system] Shanghai kou qiang yi xue $=$ Shanghai journal of stomatology. 2012;21:62-65.

42. Thumati P, Manwani R, Mahantshetty M. The effect of reduced disocclusion time in the treatment of myofascial pain dysfunction syndrome using immediate complete anterior guidance development protocol monitored by digital analysis of occlusion. Cranio: the journal of craniomandibular practice. 2014;32:289-299

43. Ma FF, Hu XL, Li JH, Lin Y. [Normal occlusion study: using T-Scan III occlusal analysis system] Zhonghua kou qiang yi xue za zhi=Zhonghua kouqiang yixue zazhi=Chinese journal of stomatology. 2013;48:363-367.

44. Abdelnabi MH, Swelem AA, Al-Dharrab AA. Influence of denture adhesives on occlusion and disocclusion times. $\mathrm{J}$ Prosthet Dent. 2016;115:306-312.

45. Makofsky HW, Sexton TR, Diamond DZ, Sexton MT. The effect of head posture on muscle contact position using the T-Scan system of occlusal analysis. Cranio: the journal of craniomandibular practice. 1991;9:316-321.

46. Kerstein RB. Disclusion time measurement studies: A comparison of disocclusion time between chronic myofascial pain dyfunction patients and nonpatients: A population analysis. J Prosthet Dent. 1994;72:473-480. 